\title{
Influência da presença do bezerro no momento da ordenha sobre o desempenho reprodutivo de vacas mestiças Holandês-Zebu
}

[Effect of calf presence during milking on reproductive performance of Holstein $x$ Zebu crossbred cows]

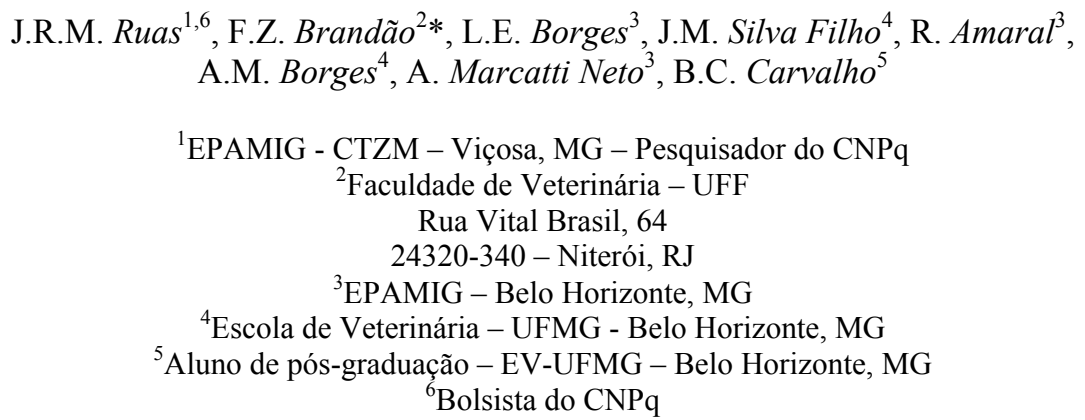

\section{RESUMO}

Avaliou-se a influência da presença do bezerro no momento da ordenha sobre os parâmetros reprodutivos de 75 vacas primíparas e multíparas mestiças Holandês-Zebu, divididas em três grupos experimentais: GI - vacas ordenhadas sem a presença do bezerro; GII - vacas ordenhadas com a presença momentânea dos bezerros, sendo estes retirados da sala de ordenha logo após a descida do leite; e GIII - vacas ordenhadas com a presença constante dos bezerros, sendo estes amarrados ao lado da vaca durante toda a ordenha. $\mathrm{O}$ escore médio da condição corporal ao parto foi de 3,1. O período de serviço, dias para o retorno ao cio e as taxas de manifestação de cio e gestação até os 120 dias pós-parto não foram influenciados $(\mathrm{P}>0,05)$ pelo tipo de manejo de ordenha.

Palavras-chave: vaca, presença da cria, período de serviço

\begin{abstract}
This study was carried out to evaluate the effect of calf presence in the milking parlour on the reproductive performance of seventy five crossbred (Holstein $\times$ Zebu) cows randomly allocated into three groups: group I - cows milked without the calf presence; group II - cows mantained with their calves during short period of time before milking to promote milk ejection and thereafter the calves were put out the milking room until the cows had finished milking; and group III - cows mantained with the calf during milking. The average body condition score at calving was 3.1. The service period, number of days to return to estrous and estrous and pregnant rates until 120 day postpartum were not influenced $(P>0.05)$ by milking management of cows. The calf presence in the milking parlour did not influence the reproductive performance of Holandês-Zebu crossbred cows.
\end{abstract}

Keywords: cow, calf presence, service period, crossbred

Recebido em 24 de novembro de 2004

Aceito em 18 de abril de 2006

Autor para correspondência (corresponding author)

E-mail: fzbr@vm.uff.br

Apoio: FAPEMIG 


\section{INTRODUÇÃO}

A amamentação restrita é característica dos sistemas de criação de bovinos de duplo propósito, baseada na produção de leite e de bezerros de corte para comercialização. Esse sistema se caracteriza pelo contato do bezerro com a mãe ou com outra vaca por período de tempo restrito antes e após a ordenha, e é amplamente utilizado nos países tropicais e subtropicais (Ugarte e Preston 1972; Campos et al., 1993; Das et al., 1999; Combellas et al., 2003).

O efeito da amamentação sobre a condição anovulatória da vaca é um dos grandes problemas dos sistemas de produção que envolvem animais de duplo propósito, em todo o mundo (Short et al. 1990). Durante mais de meio século, acreditava-se ser o estímulo das mamadas a causa do bloqueio da ovulação durante o período da lactação (McVey e Williams, 1991). Entretanto, Williams et al. (1993) concluíram que os estímulos somatossensoriais no teto e no úbere não desempenham papel na supressão da secreção do LH. Segundo Silveira et al. (1993), a criação de vínculo afetivo da vaca, aliada à interação física do bezerro na região inguinal (cabeçadas, amamentação) eram os fatores responsáveis por alterações neuroendócrinas que originam o estado anovulatório. Dentre essas mudanças, destacam-se o aumento da sensibilidade do hipotálamo ao feedback negativo do estrógeno e aumento dos estímulos dos opióides que suprimem a secreção de GnRH e LH (Griffith e Williams, 1996).

Dentre os opióides, destacam-se as endorfinas e as encefalinas, sendo a $\beta$ endorfina a mais potente de todas. Esses opióides endógenos inibem a liberação de GnRH por atuar diretamente sobre os neurônios produtores de GnRH, além de agirem na hipófise inibindo a liberação de LH (Griffith e Williams, 1996).

A habilidade das vacas amamentando em desbloquearem a ação dos opióides endógenos, durante o início do pós-parto, depende do nível de ingestão de energia e proteína a que estão submetidas. Segundo Sinclair et al. (1995), vacas amamentando e com dietas contendo altos teores de energia no início do pós-parto são submetidas por menor tempo à ação bloqueadora dos opióides endógenos sobre a secreção de LH. Assim, voltam a ciclar mais rapidamente, quando comparadas àquelas que recebem dietas com baixos teores de energia no início do pós-parto. Parece que a secreção de LH é inibida também por outros fatores que não os opióides endógenos e que as reservas de LH são influenciadas pela condição nutricional do animal.

O objetivo deste experimento foi estudar a influência da presença do bezerro no momento da ordenha de vacas mestiças Holandês-Zebu sobre o retorno ao estro após o parto, período de serviço, taxa de gestação e manifestação do cio até 120 dias pós-parto.

\section{MATERIAL E MÉTODOS}

O período experimental compreendeu o intervalo entre os meses de março de 2002 e setembro de 2003.

Durante o experimento, utilizou-se um total de 75 vacas mestiças Holandês-Zebu primíparas e multíparas de graus de sangue variado, sendo as ordens de parto e os graus de sangue distribuídos aleatoriamente entre os tratamentos, conforme Tab. 1.

Tabela 1. Ordem de partos e graus de sangue das vacas

\begin{tabular}{lccc}
\hline Ordem de parto & Grupo I & Grupo II & Grupo III \\
\hline Primeiro parto & 04 & 06 & 05 \\
Segundo parto & 07 & 08 & 08 \\
Terceiro parto & 02 & 01 & 02 \\
Quarto parto ou mais & 12 & 10 & 10 \\
\hline Graus de sangue & Grupo I & Grupo II & Grupo III \\
\hline 1/2 holandês X 1/2 zebu & 14 & 11 & 09 \\
3/4 holandês X 1/4 zebu & 05 & 07 & 11 \\
5/8 holandês X 3/8 zebu & 03 & 02 & 04 \\
7/8 holandês X 1/8 zebu & 03 & 05 & 01 \\
\hline
\end{tabular}

Os animais foram distribuídos, por meio de sorteio, à medida que pariam, em três grupos experimentais com igual número de repetições, envolvendo o manejo dos bezerros na sala de ordenha: $\mathrm{GI}=$ vacas ordenhadas sem a presença do bezerro $(n=25)$; GII = vacas ordenhadas com a presença momentânea do bezerro $(n=25)$; e GIII = vacas ordenhadas com a presença constante do bezerro $(n=25)$. 
As vacas do grupo I foram ordenhadas separadamente sem a presença das crias, de forma a evitar que a presença dos bezerros dos outros grupos interferisse na ordenha. As vacas do grupo II foram também ordenhadas separadamente, porém com a presença momentânea do bezerro, que apenas foi levado à vaca para promover a descida do leite; quando a ordenha foi iniciada, esse foi retirado da sala. As vacas do grupo III foram ordenhadas com a presença constante do bezerro, ou seja, o bezerro foi levado à vaca para promover a descida do leite e, iniciada a ordenha, o bezerro permaneceu amarrado ao lado da vaca até o seu final.

As vacas foram mantidas, no período da estação chuvosa, em pastagens formadas por capim braquiária (Brachiaria decumbens e Brachiaria brizantha) e sal mineral fornecido à vontade em cochos cobertos. Os animais receberam diariamente, no momento da ordenha, em cochos individualizados, concentrado contendo $22 \%$ de proteína bruta e $75 \%$ de NDT, de acordo com a produção: para cada $3 \mathrm{~kg}$ de leite produzidos, acima dos primeiros $8 \mathrm{~kg}$ de leite, era ofertado $1 \mathrm{~kg}$ de concentrado. Na estação da seca, os animais receberam silagem de milho (Zea mays, Z.) ad libitum como fonte de volumoso, e o concentrado era ofertado na mesma relação anterior, porém após os primeiros $5 \mathrm{~kg}$ de leite produzidos.

Foram analisados o peso, a condição corporal das vacas (ao parto e no $1^{\circ}$ cio - parâmetros de controle) e as características reprodutivas (dias para retorno ao estro pós-parto, período de serviço, taxa de gestação e de manifestação de cio até 120 dias pós-parto). Foram realizadas pesagens e avaliações do escore corporal a cada 28 dias, a partir da data do parto. A avaliação do escore corporal seguiu a escala de 3 a 5 pontos (3- baixo e 5 - alto; citada por Ruas, 1998). Os parâmetros reprodutivos foram avaliados com base no dia da manifestação do estro após o parto e no número de animais gestantes após cobrições realizadas por monta natural. A observação do estro foi realizada após a realização de cada ordenha.

Para peso corporal ao parto e ao primeiro cio (parâmetros de controle) e as demais variáveis (escore corporal, dias para retorno ao estro e período de serviço), foi utilizado o teste KruskalWallis, por se tratarem de dados qualitativos e por não apresentarem uma distribuição normal. Os dados proporcionais (taxa de manifestação e taxa de gestação) foram submetidos ao teste de qui-quadrado (Snedcor e Cochran, 1980). Para o processamento das análises, utilizou-se o programa SAS (User's..., 1996) e o programa InStat 3.

Modelo estatístico:

$\mathrm{Y}_{\mathrm{ijk}}=\mathrm{M}+\mathrm{P}_{\mathrm{i}}+\mathrm{E}_{\mathrm{ijk}}$; em que:

$\mathrm{Y}_{\mathrm{ijk}}=$ observação do manejo do bezerro na sala de ordenha na repetição k;

$\mathrm{M}=$ efeito médio geral;

$\mathrm{P}_{\mathrm{i}}=$ efeito do manejo do bezerro na sala de ordenha i ( $\mathrm{i}=$ animais ordenhados sem bezerro ou ordenhados com presença constante ou momentânea);

$\mathrm{E}_{\mathrm{ijk}}=$ erro aleatório da observação do manejo do bezerro na sala de ordenha i na repetição $\mathrm{k}$.

\section{RESULTADOS E DISCUSSÃO}

$\mathrm{Na}$ Tab. 2 encontram-se os valores para peso e escore da condição corporal dos animais no início do experimento, verificando-se uma homogeneidade $(\mathrm{P}>0,05)$ entre os grupos experimentais para as duas características.

Tabela 2. Peso médio ( $\mathrm{kg}$ ) e escore corporal (escala 3 a 5) ao parto de vacas mestiças Holandês-Zebu submetidas a diferentes manejos das crias durante a ordenha

\begin{tabular}{lcccc}
\hline \multirow{2}{*}{ Grupos experimentais } & \multirow{2}{*}{$\mathrm{n}$} & Peso médio $(\mathrm{kg})$ & \multicolumn{2}{c}{ Escore corporal (escala de 3 a 5) } \\
\cline { 3 - 5 } & 25 & média \pm desvio & média \pm desvio & mediana \\
\hline GI - ausência do bezerro & 25 & $507,9 \pm 65,9$ & $3,1 \pm 0,7$ & 3,0 \\
GII - presença momentânea do bezerro & 25,0 & $3,0 \pm 0,6$ & 3,0 \\
GIII - presença constante do bezerro & 25 & $494,6 \pm 76,8$ & $3,1 \pm 0,4$ & 3,0 \\
\hline
\end{tabular}


$\mathrm{Na}$ Tab. 3 estão apresentados os dados referentes ao período (dias) para o retorno ao cio pós-parto. A adoção de diferentes manejos das crias durante a ordenha não influenciou $(\mathrm{P}>0,05)$ o retorno à atividade ovariana luteal cíclica (AOLC) no pósparto. Yavas e Walton (2000) relataram que o principal objetivo na pecuária é a produção de um bezerro por ano, e que, para ser alcançado, o produtor dispõe de período de 85 dias após o parto para que este animal se torne gestante. Neste experimento, esse objetivo não foi possível de ser alcançado, pois o cio só foi observado a partir de 100 dias após o parto.
As taxas de manifestação de cio até 120 dias pósparto nos animais dos diferentes grupos experimentais estão apresentadas na Tab. 4, não se observando diferenças entre elas $(\mathrm{P}>0,05)$. Entretanto, ao considerarmos um nível de significância de $0,06 \%$, no tratamento sem bezerro, houve maior incidência no aparecimento de cio nos primeiros 120 dias pós-parto, quando comparado com os animais que tiveram a presença constante do bezerro na sala de ordenha.

Para as variáveis peso e escore da condição corporal ao primeiro cio observado, não houve diferença $(\mathrm{P}>0,05)$ entre os grupos (Tab. 5).

Tabela 3. Dias para o retorno ao cio pós-parto de vacas mestiças Holandês-Zebu submetidas a diferentes manejos das crias durante a ordenha

\begin{tabular}{lcc}
\hline Grupos experimentais & $\mathrm{n}$ & média \pm desvio (dias) \\
\hline GI - ausência do bezerro & 25 & $100,1 \pm 66,7$ \\
GII - presença momentânea do bezerro & 23 & $130,8 \pm 70,8$ \\
GIII - presença constante do bezerro & 20 & $119,8 \pm 52,6$ \\
\hline
\end{tabular}

Tabela 4. Taxa de manifestação de cio (\%) até 120 dias pós-parto, em vacas mestiças Holandês-Zebu, submetidas a diferentes manejos das crias durante a ordenha

\begin{tabular}{lcc}
\hline Grupos experimentais & animais em cio/total de animais & $\%$ \\
\hline GI - ausência do bezerro & $19 / 25$ & 76,0 \\
GII - presença momentânea do bezerro & $13 / 25$ & 52,0 \\
GIII - presença constante do bezerro & $11 / 25$ & 44,0 \\
\hline
\end{tabular}

Tabela 5. Peso médio (kg) e escore corporal (escala 3 a 5) no primeiro cio de vacas mestiças HolandêsZebu submetidas a diferentes manejos das crias durante a ordenha

\begin{tabular}{lcccc}
\hline \multirow{2}{*}{ Grupos experimentais } & $\mathrm{n}$ & Peso médio $(\mathrm{kg})$ & Escore corporal (escala de 3 a 5) & mediana \\
\cline { 3 - 5 } & & média \pm desvio & média \pm desvio & 3,0 \\
GI - ausência do bezerro & 25 & $511,4 \pm 59,9$ & $3,1 \pm 0,7$ & 3,0 \\
GII - presença momentânea do bezerro & 22 & $486,5 \pm 53,6$ & $2,9 \pm 0,4$ & 3,0 \\
GIII - presença constante do bezerro & 19 & $489,8 \pm 50,8$ & $2,9 \pm 0,4$ & \\
\hline
\end{tabular}

Todos os animais ao parto e no primeiro cio pósparto apresentaram valores do escore da condição corporal abaixo do recomendado por Ferreira (1991), que é de 3,5 a 4,0 (Tab. 2 e 5). Segundo Ferreira (1995), as principais causas de anestro no pós-parto estão associadas à baixa condição corporal ao parto. $\mathrm{O}$ autor enfatiza que boa condição corporal ao parto associada à mantença ou perda de pouco peso até 60 a 90 dias no pós-parto são condições indispensáveis para o rápido reinício da AOLC no pós-parto. Entretanto, as condições preconizadas pelo autor não foram observadas neste experimento, em que os animais pariram em baixa condição corporal (Tab. 2) e ainda perderam peso até o primeiro cio (Tab. 5).

$\mathrm{Na}$ Tab. 6 são apresentados os períodos de serviço obtidos nos grupos experimentais. A presença ou ausência dos bezerros na sala de ordenha não influenciou $(\mathrm{P}>0,05)$ o período de serviço dos animais, sendo considerado elevado em decorrência do atraso do retorno à AOLC. Esses valores se encontram acima do patamar considerado ideal, de 85 dias (Ferreira, 1991; Ferreira, 1995). 
Tabela 6. Período de serviço e taxa de anestro de vacas mestiças Holandês-Zebu submetidas a diferentes manejos das crias durante a ordenha

\begin{tabular}{lccc}
\hline \multirow{2}{*}{ Grupos experimentais } & \multicolumn{2}{c}{ Período de serviço (dias) } & \multirow{2}{*}{ Taxa de anestro (\%) } \\
\cline { 2 - 3 } & $\mathrm{n}$ & média \pm desvio & \\
\hline GI - ausência do bezerro & 24 & $110,8 \pm 66,8$ & $1(4,1 \%)$ \\
GII - presença momentânea do bezerro & 22 & $136,4 \pm 56,4$ & $3(13,6 \%)$ \\
GIII - presença constante do bezerro & 20 & $123,0 \pm 53,8$ & $5(25 \%)$ \\
\hline
\end{tabular}

O restabelecimento de ciclos estrais acompanhados por comportamento sexual após o parto é um processo gradual e influenciado por diversos fatores, dentre os quais destacam-se a nutrição e a amamentação (Jolly et al., 1995). Segundo Sinclair et al. (1995), a condição corporal do animal ao parto influencia as reservas de LH na hipófise. Wright e Russel (1990) e Yavas e Walton (2000) afirmaram que a baixa condição corporal leva à inibição dos pulsos de GnRH do hipotálamo e, conseqüentemente, à menor freqüência de pulsos de LH da hipófise. Roberts et al. (1997) e Yavas e Walton (2000) afirmaram que vacas com baixa condição corporal no pós-parto possuem maior concentração plasmática de $\mathrm{GH}$ e menores concentrações plasmáticas de insulina e IGF-1, com conseqüente redução das suas concentrações no interior dos folículos. Além disso, apresentam menor população de folículos médios e grandes nos ovários. Butler (2000) afirmou que as concentrações plasmáticas de glicose estão baixas em vacas com balanço energético negativo. $\mathrm{O}$ tecido adiposo secreta uma variedade de fatores, dentre os quais destaca-se a leptina, cuja concentração varia no sangue de acordo com a quantidade de reservas de tecido adiposo no corpo do animal. Animais com baixas concentrações de leptina têm o seu desempenho reprodutivo prejudicado, pois a sua ausência interfere com a secreção de gonadotropinas pela hipófise (Vernon et al., 2001).

Os animais pariram com baixo escore da condição corporal e perderam peso durante o pós-parto (Tab. 2 e 5). Apesar de não ter sido quantificado, mas baseando-se na literatura consultada (Wright e Russel, 1990; Sinclair et al. 1995; Butler, 2000; Yavas e Walton, 2000; Vernon et al., 2001), é provável que os animais apresentassem um perfil metabólico desfavorável para o retorno à AOLC, caracterizado provavelmente por baixas concentrações circulantes de insulina, glicose, IGF-1, leptina e altas concentrações de GH.

De acordo com Veitia e Simon (1972) e Randel (1990), a baixa condição corporal ao parto aumenta os efeitos inibitórios da amamentação sobre a AOLC, afirmativa não constatada neste trabalho. Os animais dos três grupos apresentaram baixa condição corporal ao parto e após a sua ocorrência, possivelmente, com perfil metabólico incompatível com o rápido retorno à AOLC.

Fulkerson et al. (1978), Orihuela (1990) e Margerison et al. (1994) relataram que a utilização do manejo de amamentação restrita aumentou o intervalo entre partos. Entretanto, Ferreira (1990) e Campos et al. (1993) citaram que a adoção desse manejo em vacas com boa condição corporal ao parto e submetidas a um regime alimentar adequado no pré e pós-parto, voltam a ciclar rapidamente. Desta forma, o fator limitante neste experimento parece ter sido a baixa condição corporal das vacas no pré e pósparto.

Na Tab. 7 são apresentados os dados referentes às taxas de gestação ao longo do período experimental; não houve diferenças $(P>0,05)$ entre os animais dos diferentes tratamentos. Essa observação encontra suporte no baixo número de repetições utilizado nos grupos experimentais. Amann e Pickett (1987) enfatizaram que, na experimentação animal, existe grande número de fatores que podem influenciar os resultados de um experimento e que, muitas vezes, nada tem a ver com os tratamentos estudados. Relatam ainda a sobreposição de amplitudes de respostas observadas e prováveis, quando poucos animais são estudados. Assim, os resultados do presente experimento são inconclusivos nesse aspecto. 
Influência da presença do bezerro...

Tabela 7. Taxa de gestação efetiva até 120 dias pós-parto em vacas mestiças Holandês-Zebu submetidas a diferentes manejos das crias durante a ordenha

\begin{tabular}{lcc}
\hline Grupos experimentais & animais gestantes/total de animais & $\%$ \\
\hline GI - ausência do bezerro & $17 / 25$ & 68,0 \\
GII - presença momentânea do bezerro & $10 / 25$ & 40,0 \\
GIII - presença constante do bezerro & $11 / 25$ & 44,0 \\
\hline
\end{tabular}

\section{CONCLUSÃO}

A presença do bezerro na sala de ordenha não influencia o período de serviço, retorno ao cio e as taxas de manifestação de cio e gestação até os 120 dias pós-parto de vacas mestiças HolandêsZebu.

\section{AGRADECIMENTOS}

À Empresa de Pesquisa Agropecuária de Minas Gerais (EPAMIG) e à Escola de Veterinária da UFMG.

\section{REFERÊNCIAS BIBLIOGRÁFICAS}

AMANN, R.P.; PICKETT, B.W. Principles of cryopreservation and a review of cryopreservation of stallion spermatozoa. Equine Vet. Sci., v.7, p.145-173, 1987.

BUTLER, W.R. Nutritional interactions with reproductive performance in dairy cattle. Anim. Reprod. Sci., v.60-61, p.449-457, 2000.

CAMPOS, O.F.; LIZIEIRE, R.S.; DERESZ, F. et al. Sistemas de aleitamento natural controlado ou artificial. I. Efeitos na performance de vacas mestiças holandês-zebu. Rev. Soc. Bras. Zootec., v.22, p.413-423, 1993.

COMBELlAS, J.; TESORERO, M. Cow-calf relationship during milking and its effect on milk yield and calf live weight gain. Liv. Res. Rural Dev., v.3, p.1-9, 2003.

DAS, S.M.; WIKTORSON, H.; FORSBERG, M. Effects of calf management and level of feed supplementation on milk yield and calf growth of zebu and crossbreed cattle in the semi-arid tropics. Liv. Prod. Sci., v.59, p.67-75, 1999.

FERREIRA, A.M. Efeito da amamentação e do nível nutricional na atividade ovariana de vacas mestiças leiteiras. 1990. 134f. Tese (Doutorado em Zootecnia) - Universidade Federal de
Viçosa, Viçosa, MG.

FERREIRA, A.M. Manejo reprodutivo e eficiência da atividade leiteira. Coronel Pacheco: Embrapa - CNPGL, 1991, 47p. (Embrapa CNPGL. Documentos, 46).

FERREIRA, A.M. Novos conceitos sobre anestro pós-parto. In: CONGRESSO BRASILEIRO DE REPRODUÇÃO ANIMAL, 11., 1995, Belo Horizonte. Anais... Belo Horizonte: Colégio Brasileiro de Reprodução Animal, 1995. p.62-70.

FULKERSON, W.J.; HOOLEY, R.D.; FINDLAY, J.K. Improvement in milking production of first calf heifers by multiple suckling. Austr. J. Agric. Res., v.29, p.351-357, 1978.

GRIFFITH, M.K.; WILLIAMS, G.L. Roles of maternal vision and olfaction in sucklingmediated inhibition of luteinizing hormone secretion, expression of maternal selectivity, and lactational performance of beef cows. Biol. Reprod., v.54, p.761-768, 1996.

JOLLY, P.D.; McDOUGALL, S.; FITZPATRICK, L.A. Physiological effects of undernutrition on postpartum anoestrus in cows. J. Reprod. Fert., suppl. 49, p.477-492, 1995.

MARGERISON, J.K.; PRESTON, T.R.; PHILIPS, C.J.C. Restrictial suckling of dairy cattle in humid tropics. Anim. Prod., v.58, p.463473, 1994.

MCVEY, W.R.; WILLIAMS, G.L. Mechanical masking of neurosensory pathways at the calfteat interface: endocrine, reproductive and lactational features of the suckled anestrous cow. Theriogenology, v.35. p.931-941, 1991.

ORIHUELA, A. Effect of calf stimulus on the milk yield of zebu-type cattle. Appl. Anim. Behav. Sci., v.26, p.187-190, 1990.

RANDEL, R.D. Nutrition and postpartum rebreeding in cattle. J. Anim. Sci., v.68, p.853862, 1990. 
ROBERTS, A.J.; NUGENT, R.A.; KLIND, J. et al. Circulating insulin-like growth factor I, insulin-like growth factor binding proteins, growth hormone, and resumption of estrus in postpartum cows subjected to dietary energy restriction. J. Anim. Sci, v.75, p.1909-1917, 1997.

RUAS, J.R.M. Eficiência reprodutiva e perfil metabólico de vacas zebu em relação a status reprodutivo, condição corporal, amamentação diferenciada e suplementação alimentar. 1998. 107f. Tese (Doutorado em Zootecnia) Universidade de Federal de Viçosa, Viçosa, MG.

SHORT, R.E.; BELLOWS, R.A.; STAIGMILLER, R.B. et al. Phisiological mechanisms controlling anestrus and infertility in postpartum beef cattle. J. Anim. Sci, v.68, p.799-816, 1990.

SILVEIRA, P.A.; SPOON, R.A.; RYAN, D.P. et al. Evidence for maternal behavior as a requesite link in suckling-mediated anovulation in cows. Biol. Reprod., v.49, p.1338-1346, 1993.

SINCLAIR， K.D.; BROADBENT, P.J.; HUTCHINSON, J.S.M. Naloxone evokes a nutritionally dependent $\mathrm{LH}$ response in post partum beef cows but not in mid-luteal phase maiden heifers. Anim. Sci., v.61, p.219-230, 1995.

SNEDCOR, G.W.; COCHRAN, W.G. Statistical methods. Ames: Iowa State Universty, 1980. $505 \mathrm{p}$.
UGARTE, J.; PRESTON, T. R. Rearing dairy calves by restricted suckling. 1. Effect of suckling once or twicw daily on milk production and calf growth. Rev. Cub. Cienc. Agric., v.6, p.173- 182, 1972.

USER'S guide: statistical analysis system. Relase 6.11. cary, NC : SAS Istitute, 1996.

VEITIA, J.L.; SIMON, L. Effect of two restricted suckling systems of calf rearing on milk production and calf growth. Rev. Cub. Cienc. Agric., v.6, p.189-193, 1972.

VERNON, R.G.; DENIS, R.G.P.; SØRENSEN, A. Signals of adiposity. Dom. Anim. Endocrinol., v.21, p.197-214, 2001.

WILLIAMS， G.L.; MCVEY JR， W.R.; HUNTER, J.F. Mammary somatosensory pathways are not required for suckling-mediated inhibition of luteinizing hormone secretion and delay of ovulation in cows. Biol. Reprod., v.49, p.1328-1337, 1993.

WRIGHT, I.A.; RUSSEL, A.J.F. Partition of fat, body composiion and body condition score in mature cows. An. Prod., v.38, p.23-32, 1984.

YAVAS, Y.; WALTON, J.S. Postpartum acyclicity in suckled beef cows: a review. Theriogenology, v.54, p.25-55, 2000. 